\title{
Development and Evaluation of a Particle Deposition Measurement Method Based on a CCD Imaging Chip
}

\author{
Junyi Zhuang', Fei Li ${ }^{1 *}$, Feifei Dong1, Jianlin Ren², Bin Zhou' \\ ${ }^{1}$ Department of HVAC, College of Urban Construction, Nanjing Tech University, Nanjing, 210009, China \\ ${ }^{2}$ School of Energy and Environmental Engineering, Hebei University of Technology, Tianjin 300401, China
}

\begin{abstract}
Particle deposition on indoor surfaces has lately received great attention due to increasing concern about the effects of particle exposure on human health. In this study, we developed an easy and low-cost method of measuring the deposition amounts of different-sized particles and evaluated it through particle concentration measurement and theory calculation. First, a clean charge-coupled device (CCD) chip was exposed to air in order to receive the deposition of airborne particles. After sedimentation, images generated by the CCD of the deposited particles were analyzed, and the quantity of particles in each size range $(>2 \mu \mathrm{m})$ was identified based on the unique pixel sizes. Using a particle counter and a dust monitor, we also measured the concentrations of airborne particles with different sizes near the chip during sedimentation and compared them with the numbers of settled particles. The results show that the particle deposition can be quantified with this method and that a positive correlation exists between the deposition amounts and the particle number concentrations.
\end{abstract}

Keywords: Particle deposition; Image recognition; MATLAB; CCD.

\section{INTRODUCTION}

Aerosol particles can deposit onto indoor surfaces and onto human bodies. For indoor surface deposition, the particles can cause material damage such as soiling and chemical deterioration (Nazaroff et al., 1990) and can lead to damage in electronic equipment (Weschler et al., 1996). The contaminated surfaces also play an important role in the transmission of enteric and respiratory viral infections (Lei et $a l ., 2017)$. For body surface deposition, the particles have two possible consequences. First, these deposited particles can absorb bacterial semivolatile organic compounds (SVOCs), heavy metals, and polycyclic aromatic hydrocarbons (PAHs), which can cause adverse health risks through dermal exposure. Second, the particles deposited onto eyes and skin may cause irritation, injuries, allergic reactions, and diseases (Leonardi et al., 2012). Hence, research on the particle deposition measurement method has profound meanings.

The common methods currently used to measure the sedimentation flux of particulate matter are the fluorescence method (Truskett, 2003), neutron activation analysis (NAA) (El-Araby et al., 2011), and proton-induced X-ray emission fluorescence spectrometry (PIXE) (Heidam et al., 1999). These methods are complicated and expensive to operate.

\footnotetext{
${ }^{*}$ Corresponding author.

E-mail address: faylee@njtech.edu.cn
}

For example, the fluorescence method must first label fluorescent particles and measure the amount of fluorescence. Both NAA and PIXE have high requirements for analytical materials and special large instruments. These technologies are unsuitable for daily real-time monitoring such as the settlement on indoor and human body surfaces. Details of the methods to measure particle depositions are in Lai (2010).

In the past two decades, CCD and CMOS chips have been widely used in photography. They use photosensitive elements as the basic means to capture images (Pryor et al., 1997). The image sensor can use photoelectric sensors to convert photoelectric signals into digital data. CCD sensors are superior to CMOS sensors in terms of sensitivity, resolution, and noise control, while CMOS sensors are characterized by lower cost, lower power consumption, and higher integration (Miazza et al., 2004). However, because of the continuous advancement of the CCD and CMOS sensor technology, the difference between the two has gradually narrowed. In the past, Manaresi et al. (2003) used a CMOS chip to conduct individual cell manipulation and detection. Löffler et al. (2011) investigated the dynamics and precise deposition of charged polymer microparticles onto a microelectronic chip. Wagner et al. (2011) used a surface electrode to generate an electric field in a programmable mode on an integrated circuit; then, they spatially selectively deposited charged particles onto the integrated circuit. Corkidi et al. (1998) and Jung and Lee (2016) used a CMOS chip microscope to count real-time bacterial colonies and other microbial colonies. In these articles, there are no direct measurements of particulate 
deposition using $\mathrm{CCD} / \mathrm{CMOS}$ chips, but they have important significance and guiding value for this study.

In this paper, a combination of the CCD chip sensor and Raspberry $\mathrm{Pi}$ is used to measure the sedimentation of particles. The images taken by the CCD chip are processed by a MATLAB imaging process code to directly obtain the size distribution of deposited particles. The results were also compared with the measured concentrations and theory calculations.

\section{METHODS}

\section{CCD Sampling System Design}

Hardware Setup

We selected a CCD chip with the image area of $2592 \mu \mathrm{m} \times$ $1944 \mu \mathrm{m}$ and the pixel size of $1.4 \mu \mathrm{m} \times 1.4 \mu \mathrm{m}$. This CCD chip was refitted from a camera (OV05647-G04A; OmniVision, USA) by removing its lens (Fig. 1). Since the CCD chip was directly exposed to the light, the color and intensity of the light source and the distance of the light source from the chip affect the imaging results. Finally, we selected soft white light, which had even output and uniform distribution. When particles deposited onto the chip, they would block the light, and the pixels with the same size as the particles would become black. By analyzing the pixel color of the chip, the number and size of settled particles could be calculated. The chip can identify particles with diameter of at least $1.4 \mu \mathrm{m}$. The images were automatically saved by a Raspberry Pi (Model B+; UK) connected with the chips.

\section{Particle Diameter and Deposition Flux Calculation}

In this study, when a solid particle deposited onto the
CCD chip, its projected area, which was the same size as the particle, could be obtained as the product of the number of blocked pixels $(n)$ and the area of each pixel $(a)$. The particle equivalent diameter $d_{p}$ is calculated from the projected area by Eq. (1):

$d_{p}=\sqrt{\frac{4 n a}{\pi}}$

The number of projections is the particle number; therefore, the particle deposition flux is calculated by Eq. (2):

$J_{c, d_{p}}=\frac{N_{c, d_{p}}}{T \cdot A}$

where $T$ is the time length for the chip exposed in the air, $A$ is the area of the chip, and $N_{c, d p}$ is number of deposited particles (projections) with diameter $d_{p}$ over period $T$. MATLAB image-processing tools were used to solve these equations.

\section{Validation for the CCD Sampling System}

For the particle deposition velocity on surfaces, Shi et al. (2016) summarized the experimental data and developed an empirical equation to compute the deposition velocity for different-sized particles. The deposition velocity $V_{T S, d p}$ can be expressed as:

$V_{T S, d_{p}}=2.89 \times\left(\log d_{p}\right)^{2}+0.43 \times\left(\log d_{p}\right)+0.12$

where $d_{p}$ is the particle diameter. The deposition velocity

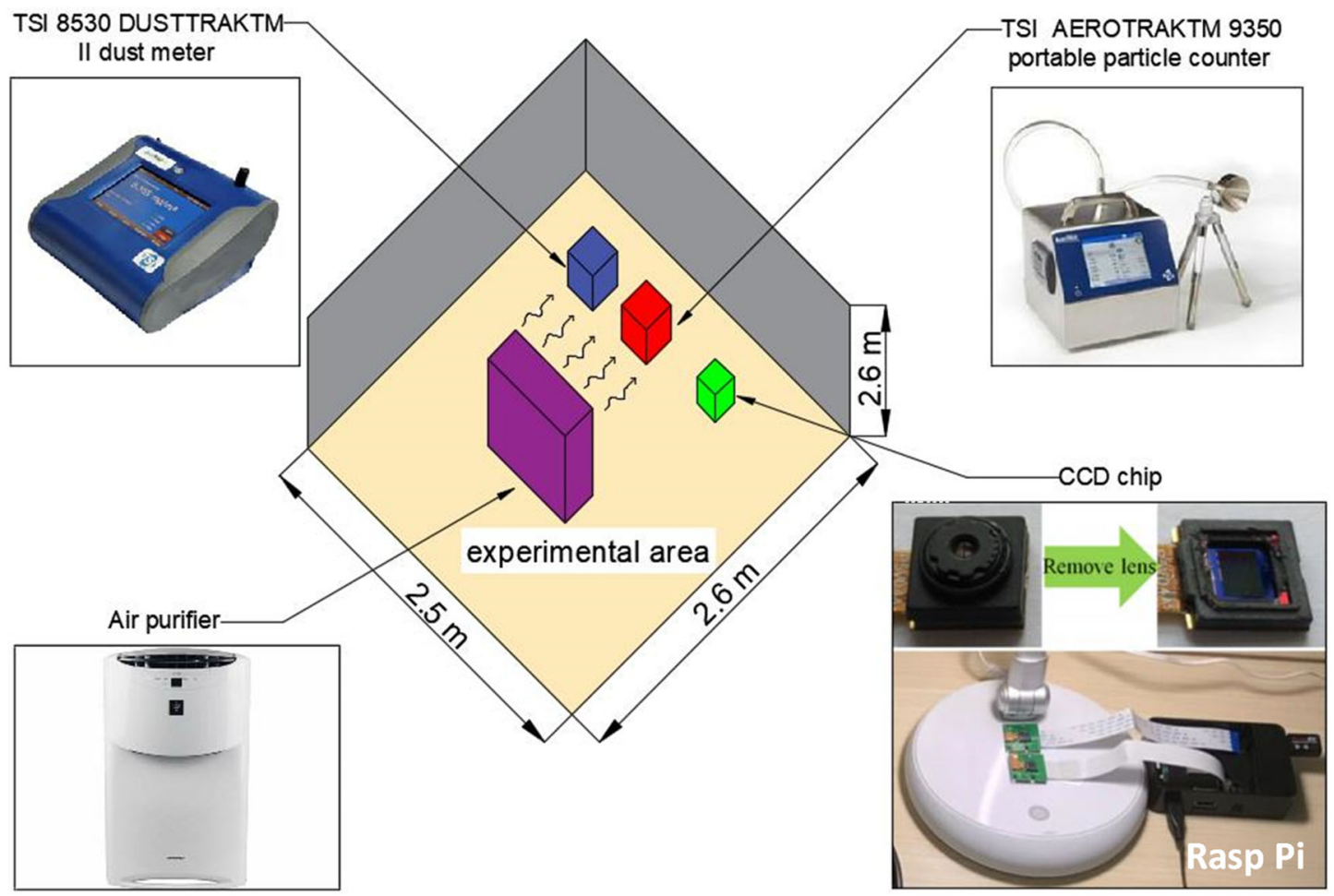

Fig. 1. Sketch map of the experimental system. 
can be used to calculate the deposited particle number. The theoretical deposited particle number is expressed as follows:

$N_{t, d p}=J_{t, d p} \cdot T \cdot A=V_{T S, d p} \cdot C_{d p} \cdot T \cdot A$

where $N_{t, d p}$ is the theoretical settlement amount for the particle with diameter $d_{p}, C_{d p}$ is the particle number concentration in the air, $T$ is the time interval length, $A$ is the CCD chip area, and $J_{t, d p}$ is the theoretical deposition flux for each diameter.

From Eq. (4), it is found that higher particle number concentration will result in more deposited particles, and this can be used to validate the CCD sampling system. In this study, an air purifier was placed next to the CCD chip to control its surrounding particle concentration (Fig. 1). The room volume was $17 \mathrm{~m}^{3}$, and the max CADR of the air purifier was $180 \mathrm{~m}^{3} \mathrm{~h}^{-1}$. The CADR was sufficiently large to control the particle concentration. We used AeroTrak 9350 (TSI Inc., USA) and DustTrak 8530 (TSI Inc., USA) to monitor the particle number concentration $(>2 \mu \mathrm{m})$ and mass concentration $\left(\mathrm{PM}_{10}\right)$.

As shown in Fig. 2, the mass and number concentrations have almost identical tendencies, and four time intervals with different concentrations were selected. The sampling time for each time interval was an important parameter. If the sampling time was short, the number of deposited particles on the chip was small and had no statistical significance. And if the sampling time was too long, the time resolution would be too low. In this experiment, 10 hours was selected as the sampling time. In each time interval, the confidence intervals of particle number and mass concentrations were calculated, and the size distributions of the particles deposited onto the chip under three different light intensities were also analyzed using the aforementioned method. The particle deposition and average concentration data for each time interval are compared in the "Results" section in this paper.

\section{RESULTS}

\section{CCD Chip Sampling}

For each time interval, two images were recorded: one was captured at the beginning of the interval, and the other was captured at the end of the interval. Since most of particles are solid in air environment and overlap of deposited particles is rare, the sizes of identical particles do not change during the sampling time (Fig. 3). By comparing these two figures, we obtained the size distribution of the settled particles during this time interval. Fig. 3 shows the pair of images in Time Interval $2(35-45 \mathrm{~h})$ and their reconstructions. These two images were only small fractions of their originals due to their large sizes. Based on Fig. 3 and Eq. (1), a custom MATLAB program was used to analyze the size distribution of deposited particles larger than $2 \mu \mathrm{m}$ within 10 hours. In Time Interval 2, for the diameters of $2-3 \mu \mathrm{m}, 3-5 \mu \mathrm{m}$, and $>5 \mu \mathrm{m}$, the averaged deposited particle number on the chip was 23,28 , and 6 , respectively.

In addition, $\mathrm{CCD}$ chip may have static electricity which can affect the particle deposition (Feng et al., 2016; Feng and Cao, 2019). As shown in Fig. 1, we used two CCD sensors in the experiment. One CCD sensor was powered on during the sampling period, while the other CCD sensor was powered off. Comparing the number of deposited particles on these two CCD chips, the difference is not obvious, which means static electricity does not have an obvious effect on the performance of the sampling system.

\section{Comparison with the Measured Concentration}

Fig. 4 shows the number of deposited particles measured by $\mathrm{CCD}$ chips and the averaged mass concentration $\left(\mathrm{PM}_{10}\right)$ measured by DustTrak 8530 at different time intervals. The error bars in the figure represent $95 \%$ confidence intervals. The error of the number of deposited particles was mainly induced by different light intensities for CCD imaging, and the error of the mass concentration mainly resulted from the concentration fluctuations (Fig. 2). As shown in Fig. 4, Time Interval 2 had the highest mass concentration followed by Time Interval 1, and Time Interval 3 had the smallest mass concentration. The distributions of the settled particle number during different time intervals were similar to those of the averaged mass concentrations. When the averaged mass concentration $\left(\mathrm{PM}_{10}\right)$ increased, the number of settled

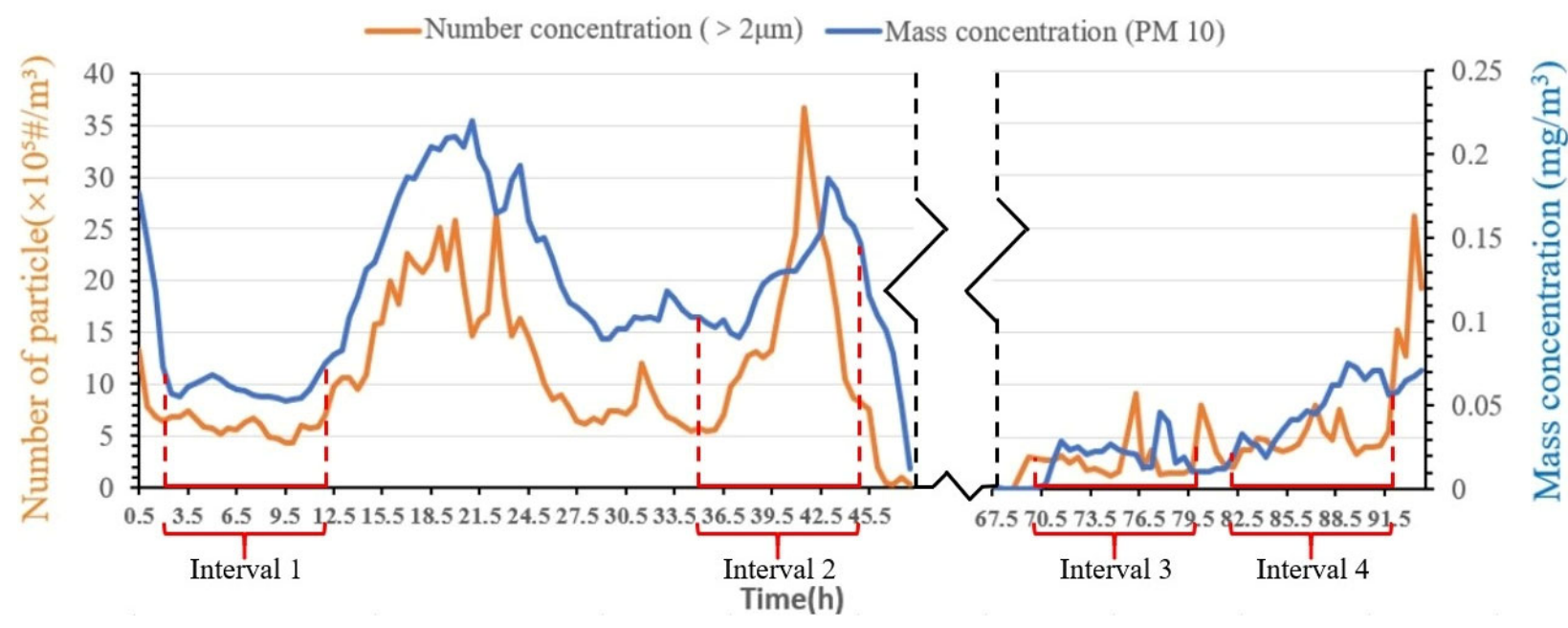

Fig. 2. Sampling particle number and mass concentration. 
(a) Time-series images

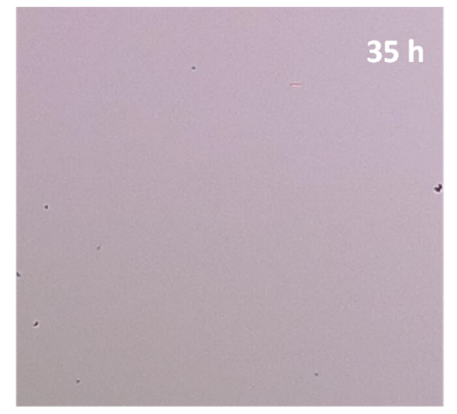

(b) Reconstructed images

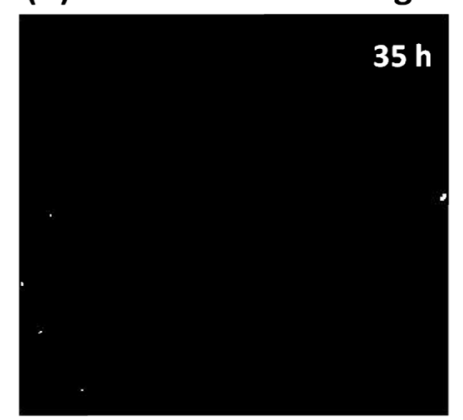

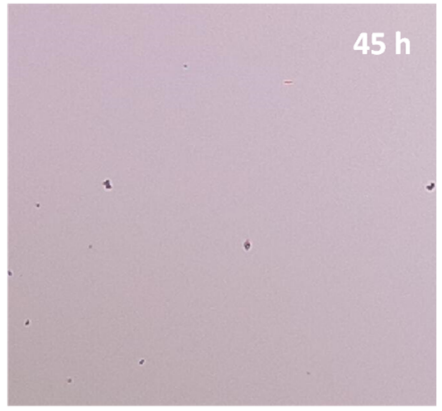

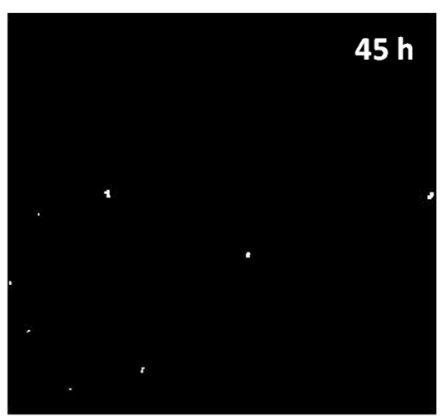

Fig. 3. Image processing: (a) time-series images of Time Interval 2; (b) reconstruction images using a custom MATLAB program.

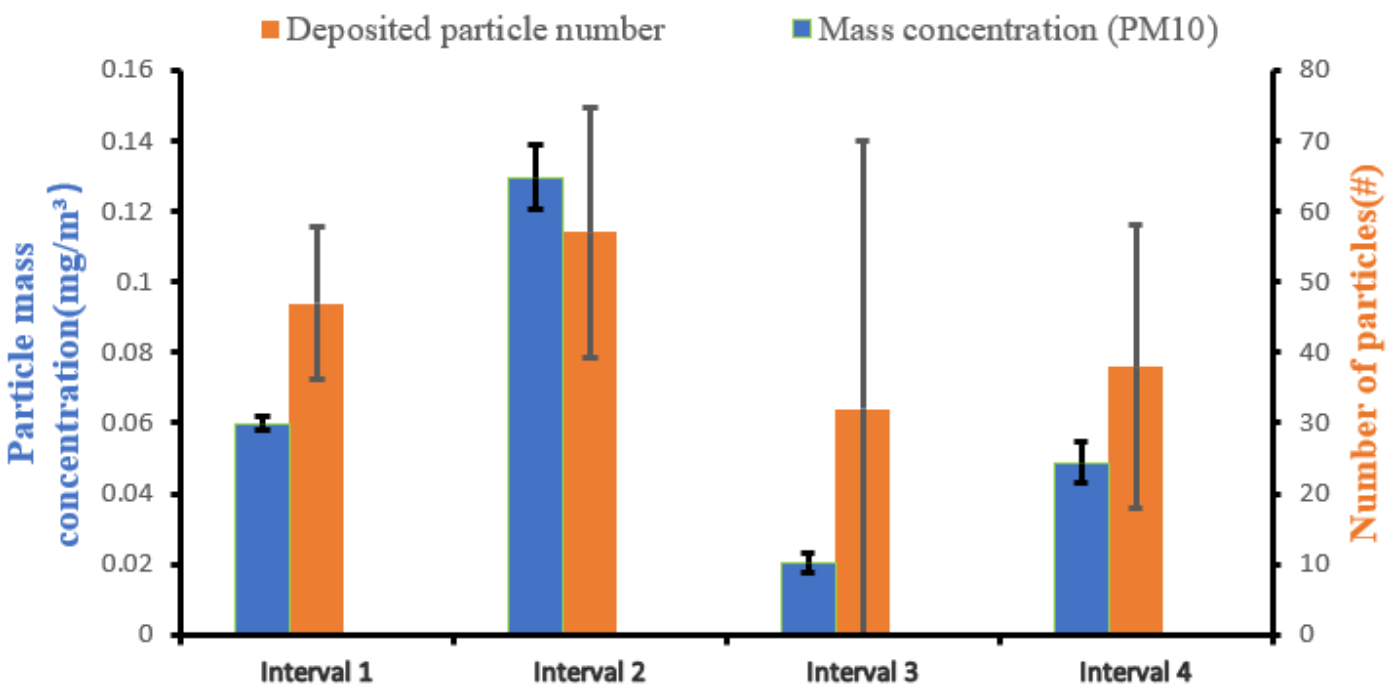

Fig. 4. Comparison of the deposited particle number and $\mathrm{PM}_{10}$.

particles increased and vice versa. There is a positive relationship between the averaged mass concentration and the number of deposited particles, and the particle deposition amount measured by the CCD chips can reflect the particle concentration in the air. Fig. 4 also shows that the variance of the deposited particle number is higher than that of the mass concentration especially for Time Interval 3 . This is because the CCD chip area was very small $(2592 \mu \mathrm{m} \times 1944 \mu \mathrm{m})$, fewer particles deposited on the chip within 10 hours, and this process is very stochastic. This higher uncertainty led to a larger confidence interval for the deposited particle number in Time Interval 3.

\section{DISCUSSION}

Table 1 shows the particle number concentrations measured by TSI 9350 and deposited particle numbers from the CCD chip and theory calculation. The brackets in Table 1 indicate the range of the number of particles within $95 \%$ confidence. From Table 1, Interval 2 has the largest particle number concentration in the air due to its largest mass concentration, and the deposited particle numbers obtained by the CCD and theory calculation are larger than those in the other intervals. This result confirms that the concentration of particulate pollutants in the air is proportional to the 
Table 1. Particle number for different time intervals (95\% confidence).

\begin{tabular}{llllll}
\hline Number concentration from TSI 9350 & & Interval 1 & Interval 2 & Interval 3 & Interval 4 \\
\cline { 2 - 5 }$\left(\geq 2 \mu \mathrm{m}, 10^{5} \mathrm{~m}^{-3}\right)$ & & {$[5.50,6.28]$} & {$[11.2,19.0]$} & {$[2.00,3.92]$} & {$[3.94,6.38]$} \\
\hline $\begin{array}{l}\text { Deposited particle number from CCD } \\
\text { (\#) }\end{array}$ & $2-3 \mu \mathrm{m}$ & {$\left[0^{*}, 16\right]$} & {$[11,35]$} & {$\left[0^{*}, 22\right]$} & {$\left[0^{*}, 35\right]$} \\
& $3-5 \mu \mathrm{m}$ & {$[5,15]$} & {$[18,38]$} & {$\left[0^{*}, 39\right]$} & {$[19,29]$} \\
& $>5 \mu \mathrm{m}$ & {$[25,33]$} & {$\left[0^{*}, 14\right]$} & {$[16,32]$} & {$\left[0^{*}, 3\right]$} \\
Deposited particle number from & total & {$[36,58]$} & {$[39,75]$} & {$\left[0^{*}, 70\right]$} & {$[18,58]$} \\
theory (\#) & $2-3 \mu \mathrm{m}$ & {$[21,23]$} & {$[40,70]$} & {$[7,13]$} & {$[16,26]$} \\
& $3-5 \mu \mathrm{m}$ & {$[16,20]$} & {$[24,44]$} & {$[5,13]$} & {$[6,10]$} \\
& $>5 \mu \mathrm{m}$ & {$[3,5]$} & {$[5,9]$} & {$[2,6]$} & {$[1,3]$} \\
& total & {$[43,47]$} & {$[78,114]$} & {$[18,28]$} & {$[26,36]$} \\
\hline
\end{tabular}

$0^{*}$ represents a negative value for this number due to the large confidence interval.

number of settled particles. For the theoretical calculation results, the confidence interval of Interval 1 is relatively small, followed by Interval 3 and Interval 4. Interval 2 is the largest due to its large concentration fluctuation (Fig. 2). For the CCD measurement results, Interval 3 and Interval 4 have larger confidence intervals than Interval 1 and Interval 2. This is mainly induced by the statistical uncertainty.

Table 1 also shows the differences in deposited particle numbers obtained by $\mathrm{CCD}$ and theoretical calculation. Especially for small particles with the diameter of 2-3 $\mu \mathrm{m}$, the CCD-measured number is smaller than the theoretical calculation. Since the chip resolution in the experiment is not sufficiently high $(1.4 \mu \mathrm{m} \times 1.4 \mu \mathrm{m})$, the identification capacity for small particles of $2-3 \mu \mathrm{m}$ was weak. The concentration of particulate contaminant in the air fluctuates in the 10-hour time interval, and the sedimentation of particulate matter on the small chip is slightly random. These factors also result in the deviation from theoretical calculations.

In addition, the deposition measurement approach based on the CCD chip has limitations. First, this method can only identify particles with diameters larger than $2 \mu \mathrm{m}$. Smaller particles cannot be distinguished through CCD imaging. With respect to dealing with smaller particles $(<2 \mu \mathrm{m})$, maybe using a higher-resolution CCD chip can identify the smaller particles. Second, although the soft white light used has even output and uniform distribution, its intensity has small variations. Therefore, for each image, we calculated the standard variation of the particle number under different light intensities. Third, because the CCD chip is very small, the particle deposition onto this small area is stochastic. In future studies, further verification is needed in terms of the repeatability and stability. Sampling data from several chips should be compared with those directly counted through the microscope. Besides, the indoor humidity may affect the particle concentration (Feng et al., 2018), and service life of the CCD sensor, which is exposed in the air and captures particles directly for a long time. It is indispensable that some research should be conducted to test its service life and performance under different humidity.

\section{CONCLUSIONS}

In this study, we developed and evaluated a simple method of measuring particle deposition. We used a CCD chip without a lens and MATLAB image processing code to analyze the size distributions of deposited particles. The results were then compared with the measured airborne concentrations and theory calculations. The deposited particle numbers obtained by using the CCD chip positively correlated with the measured airborne particle concentrations, i.e., higher particle concentrations in the air resulted in more observable deposited particles and vice versa. In addition, the amounts of deposition measured using the chip displayed a trend similar to our calculations, but quantitative differences remained due to the low resolution of the chip and uncertainty in the deposition.

\section{ACKNOWLEDGEMENTS}

This study was supported by the National Natural Science Foundation of China (Grant No. 51708286). The study has also been supported by the Natural Science Foundation of Jiangsu Province (Grant No. BK20171015) and China Postdoctoral Science Foundation (Grant No. 2019M651818).

\section{REFERENCES}

Corkidi, G., Diaz-Uribe, R., Folch-Mallol, J.L. and NietoSotelo, J. (1998). Covasiam: An image analysis method that allows detection of confluent microbial colonies and colonies of various sizes for automated counting. Appl. Environ. Microbiol. 64: 1400-1404.

El-Araby, E.H., Abd El-Wahab, M., Diab, H.M., EiDesouky, T.M. and Mohsen, M. (2011). Assessment of atmospheric heavy metal deposition in north egypt aerosols using neutron activation analysis and optical emission inductively coupled plasma. Appl. Radiat. Isot. 69: 1506-1511.

Feng, Z. and Cao, S.J. (2019). A newly developed electrostatic enhanced pleated air filters towards the improvement of energy and filtration efficiency. Sustainable Cities Soc. 49: 101569.

Feng, Z., Long, Z. and Yu, T. (2016). Filtration characteristics of fibrous filter following an electrostatic precipitator. J. Electrostat. 83: 52-62.

Feng, Z., Zhou, X., Xu, S., Ding, J. and Cao, S.J. (2018). Impacts of humidification process on indoor thermal comfort and air quality using portable ultrasonic humidifier. Build. Environ. 133: 62-72. 
Heidam, N.Z., Wahlin, P. and Christensen, J.H. (1999). Tropospheric gases and aerosols in northeast greenland. J. Atmos. Sci. 56: 261-278.

Jung, J.H. and Lee, J.E. (2016). Real-time bacterial microcolony counting using on-chip microscopy. Sci. Rep. 6: 21473.

Lai, A.C. (2010). Particle deposition indoors: A review. Indoor Air 12: 211-214.

Lei, H., Li, Y., Xiao, S., Yang, X., Lin, C., Norris, S.L., Wei, D., Hu, Z. and Ji, S. (2017). Logistic growth of a surface contamination network and its role in disease spread. Sci. Rep. 7: 14826.

Leonardi, A., Bogacka, E., Fauquert, J.L., Kowalski, M.L., Groblewska, A., Jedrzejczak-Czechowicz, M., Doan, S., Marmouz, F., Demoly, P. and Delgado, L. (2012). Ocular allergy: Recognizing and diagnosing hypersensitivity disorders of the ocular surface. Allergy 67: 1327-1337.

Löffler, F., Wagner, J., König, K., Märkle, F., Fernandez, S., Schirwitz, C., Torralba, G., Hausmann, M., Lindenstruth, V. and Bischoff, F.R. (2011). High-precision combinatorial deposition of micro particle patterns on a microelectronic chip. Aerosol Sci. Technol. 45: 65-74.

Manaresi, N., Romani, A., Medoro, G. and Altomare, L. (2003). In IEEE International Solid-state Circuits Conference, 2003.

Miazza, C., Dunand, S., Wyrsch, N., Shah, A., Blanc, N., Kaufmann, R. and Cavalier, L. (2004). Performance analysis of a-si:H detectors deposited on cmos chips. MRS. Proc. 808: A4.46.

Nazaroff, W.W., Ligocki, M.P., Ma, T. and Cass, G.R. (1990). Particle deposition in museums - comparison of modeling and measurement results. Aerosol Sci. Technol. 13: $332-348$.

Pryor, W.R., Na, C.Y., Cochran, A.L., Cochran, W.D., Barker, E.S., Armosky, B.J. and Pulliam, C.E. (1997). Mcdonald observatory data on the comet shoemaker-levy 9 impacts on jupiter and the resulting haze particles. Planet. Space Sci. 45: 1299-1313.

Shi, S., Chen, C. and Zhao, B. (2016). Modifications of exposure to ambient particulate matter: Tackling bias in using ambient concentration as surrogate with particle infiltration factor and ambient exposure factor. Environ. Pollut. 94: 1024-1029.

Truskett, V.N. (2003). Influence of surfactants on an evaporating drop: Fluorescence images and particle deposition patterns. Langmuir 19: 8271-8279.

Wagner, J., König, K., Förtsch, T., Löffler, F., Fernandez, S., Felgenhauer, T., Painke, F., Torralba, G., Lindenstruth, V., Stadler, V., Bischoff, F.R., Breitling, F., Hausmann, M. and Nesterov-Müller, A. (2011). Microparticle transfer onto pixel electrodes of $45 \mu \mathrm{m}$ pitch on hv-cmos chipssimulation and experiment. Sens. Actuators, A 172: 533545.

Weschler, C., Shields, H. and Shah, B. (1996). Understanding and reducing the indoor concentration of submicron particles at a commercial building in southern california. J. Air Waste Manage. Assoc. 46: 291-299.

Received for review, May 7, 2019

Revised, August 7, 2019

Accepted, September 21, 2019 